Review began 09/10/2021 Review ended 09/15/2021 Published 09/18/2021

๑) Copyright 2021

Pina Cabral et al. This is an open access article distributed under the terms of the Creative Commons Attribution License CCBY 4.0., which permits unrestricted use, distribution, and reproduction in any medium, provided the original author and source are credited.

\section{Spontaneous Tumor Lysis Syndrome in Prostate Cancer}

\author{
João Pina Cabral ${ }^{1}$, Joana Coelho ${ }^{1}$, Jorge Fortuna ${ }^{1}$, Adriano Rodrigues ${ }^{1}$ \\ 1. Internal Medicine, Centro Hospitalar e Universitário de Coimbra, Coimbra, PRT
}

Corresponding author: João Pina Cabral, joao@pinacabral.com

\begin{abstract}
Tumor lysis syndrome (TLS) is an oncological emergency that most frequently occurs in hematological and high-turnover solid neoplasia. Its incidence in solid, slowly proliferating neoplasia is unclear, primarily because of few published case reports. TLS may be triggered by chemotherapy or infection, or may spontaneously arise. Here, we present a review of the literature and a case of a 58-year-old male patient with prostate cancer who developed spontaneous TLS.
\end{abstract}

Categories: Internal Medicine, Urology, Oncology

Keywords: tumor lysis syndrome, prostate cancer, solid tumor, low turnover, oncologic emergency, spontaneous tumor lysis syndrome

\section{Introduction}

Tumor lysis syndrome (TLS), which is considered an oncological emergency, is characterized by hyperuricemia, hyperkalemia, hyperphosphatemia, and hypocalcemia [1]. TLS occurs more frequently in hematological and fast-growing bulky neoplasia, although it is rare in solid tumors, and spontaneous cases are less common. The prognosis of TLS associated with solid tumors is worse than when present in hematological neoplasia [2]. Here, we report a case of TLS in a 58-year-old man with prostate cancer. We describe how we diagnosed and treated TLS and review the current relevant literature. This case report was presented by the main author as oral communication in the Portuguese National Congress of Internal Medicine.

\section{Case Presentation}

We encountered a 58-year-old man who was admitted to our emergency department (ED) with localized right hypochondriac pain. In the ED, the patient underwent abdominal ultrasound testing, which showed multiple lesions suggesting metastatic neoplasia. He was admitted for further testing and pain management. One year earlier, the patient was diagnosed with prostate cancer (PC) with metastatic lesions in his axial skeleton and has since been treated with leuprorelin.

The patient presented with worsening pain, requiring continuously increasing doses of analgesics while he was waiting to undergo imaging procedures. Full-body computed tomography (CT) showed no evidence of a synchronous primary neoplasm, although multiple secondary hepatic lesions were detected (Figure 1). The patient's fragile condition prevented invasive biopsy for histological testing. 


\section{Cureus}

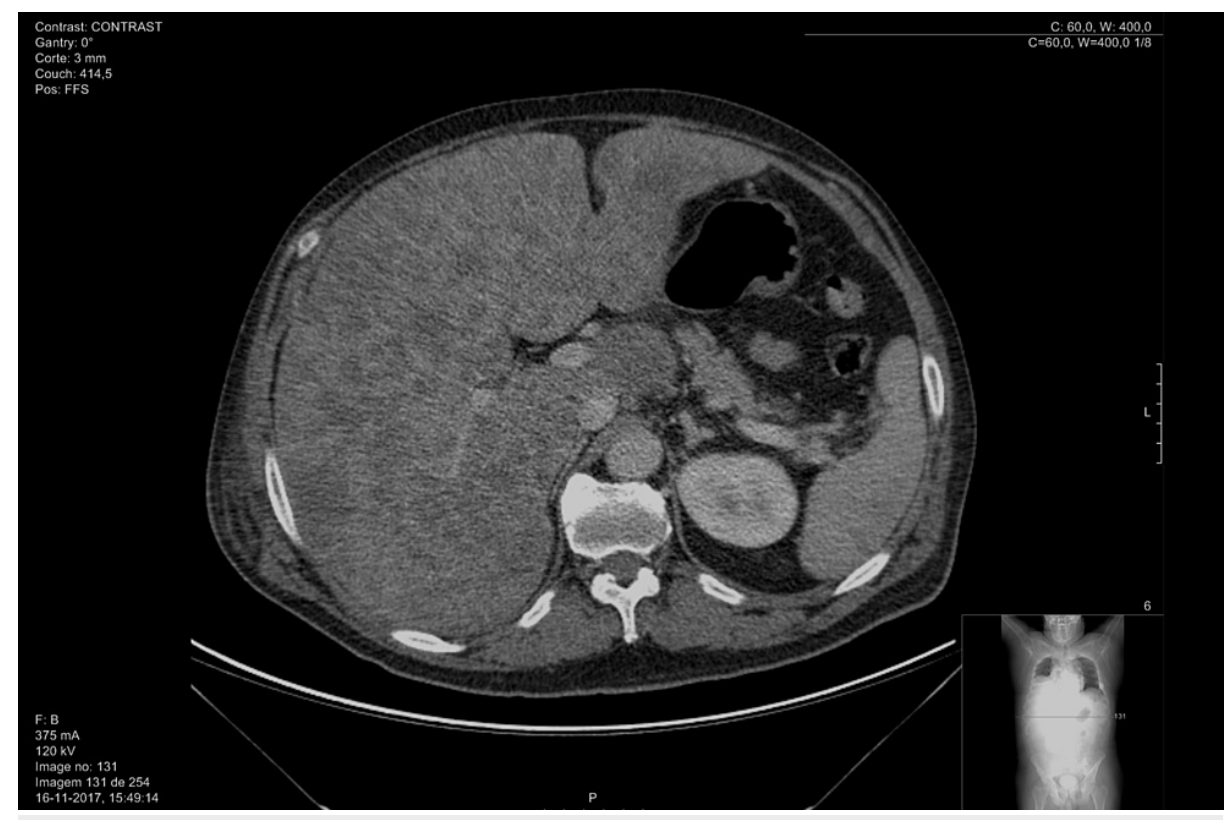

FIGURE 1: Full-body CT scan showing hepatic lesions.

On the second day of hospitalization, the patient experienced sudden neurological depression, with a Glasgow Coma Scale of 10, which prompted new blood tests (Table 1). The new onset of hyperuricemia, hyperkaliemia, hyperphosphatemia, and acute kidney failure accompanied by increased creatinine and oliguria led us to consider the diagnosis of TLS according to the revised Cairo-Bishop classification (Table 2). Fluid reposition, rasburicase, and sevelamer, along with empirical piperacillin/tazobactam, against Pseudomonas infections, were initiated. The next day, despite analytical improvement (Table 1), the patient succumbed to his illness.

\begin{tabular}{|c|c|c|c|}
\hline \multirow[b]{2}{*}{ Metabolite or electrolyte } & \multicolumn{3}{|c|}{ Blood analysis } \\
\hline & Baseline & 1st day & 2nd day \\
\hline Uric acid (mg/dL) & 4 & 15 & 1 \\
\hline Potassium (mEq/L) & 4,46 & 4.94 & 4.8 \\
\hline Phosphorus (mg/dL) & 3.84 & 8.39 & 8.05 \\
\hline Calcium (mmol/L) & 2.50 & 2.29 & 2.02 \\
\hline Creatinine $(\mu \mathrm{mol} / \mathrm{L})$ & 82 & 184.9 & 326 \\
\hline
\end{tabular}

TABLE 1: Blood analyses. 


\section{Cureus}

Laboratory tumor lysis syndrome - abnormality in two or more

Metabolite or electrolyte

Uric acid

Potassium

Phosphorus

Calcium

Clinical tumor lysis syndrome

LTLS and at least one of the following: (1) creatinine $\times 1.5$ ULN (age >12 years or age-adjusted); (2) cardiac arrhythmia or sudden death; and (3) seizure

TABLE 2: Cairo-Bishop classification for tumor lysis syndrome.

LTLS, laboratory tumor lysis syndrome; ULN, upper limit of normal.

\section{Diagnostic criteria}

$\geq 8 \mathrm{mg} / \mathrm{dL}$ or $25 \%$ increase from baseline

$\geq 6 \mathrm{mEq} / \mathrm{L}$ or $25 \%$ increase from baseline

$\geq 6.5 \mathrm{mg} / \mathrm{dL}$ (children), $\geq 4.5 \mathrm{mg} / \mathrm{dL}$ (adults), or $25 \%$ increase from baseline

$\geq 25 \%$ decrease from baseline

\section{Discussion}

TLS is an oncological emergency characterized by hyperuricemia, hyperkalemia, hyperphosphatemia, and hypocalcemia, and is caused by massive destruction of neoplastic cells [1,3]. Further, acute kidney failure is a common consequence, and metabolic acidosis may occur [4]. High-turnover hematological malignancies such as Burkitt lymphoma and acute lymphoblastic leukemia are common causes of TLS [5], and regular electrolyte and metabolite monitoring is required during treatment. TLS develops during or shortly after the initiation of therapy using glucocorticoids, hormonal agents, or chemotherapeutics such as rituximab [6]. In contrast to hematological neoplasia, solid tumors seldomly present with TLS, and the spontaneous development of the latter is exceptional [7]. Moreover, the prevalence of TLS in solid tumors is difficult to establish because of few published case reports [8]. Although the development of TLS in hematological neoplasia may be anticipated before initiating therapy, this does not apply to solid tumors [9]. For example, TLS is a rare complication of PC [1]. Mirrakhimov et al. presented a case series of TLS in solid tumors, with only five associated with PC [10].

TLS that occurs in patients with solid tumors and spontaneous TLS (STLS) in conjunction with all tumors are associated with increased mortality [7,9]. Most cells proliferate in hematological tumors, and the ensuing large cell mass harbors identical genetic abnormalities, which confer the same theoretical sensitivity to chemotherapy. In contrast, the pathophysiological mechanisms of TLS in solid tumors are not fully understood. Possible mechanisms, which initiate the rapidly ensuing inflammatory cascade, include tissue necrosis from deficient vascularization caused by rapid uncontrolled growth or increased tissue pressure (mostly on the liver and bone) [7].

Patients at risk for developing TLS should be evaluated before treatment according to analyses of the complete blood count, serum electrolytes and metabolites, and urine. Hyperuricemia may be present upon initiation of therapy, and renal deposition of uric acid is facilitated by the acidic environment produced by lactic acidosis and dehydration, leading to renal failure. A ratio of urinary uric acid to urinary creatinine $>1$ suggests urate nephropathy, whereas a ratio of $<1$ indicates other causes. Hyperphosphatemia and consequent hypocalcemia may cause renal deposition of calcium and exacerbate renal failure. Interestingly, in STLS, rapidly proliferating tumor cells reutilize released phosphorous such that hyperphosphatemia is less common than in TLS [11]. Further, the destruction of tumor cells releases high concentrations of potassium into the bloodstream. Severe renal failure may lead to diminished potassium excretion and fatal arrhythmias.

When TLS develops, the main goal is to normalize the levels of serum electrolytes and metabolites to prevent or ameliorate the severity of acute kidney failure and to prevent fatal arrhythmias. Clinical and analytical reassessment should be performed every four to six hours [11].

\section{Conclusions}

TLS and STLS delay further chemotherapy and directly or indirectly lead to increased mortality. Although infrequently associated with solid tumors, TLS must be considered when treating any patient with cancer, and close monitoring is warranted. The low incidence of TLS in solid tumors explains our insufficient knowledge of its causes, consequences, and therapeutic strategies. For example, the few published studies describe only single or a small series of cases. Further, the incidence of STLS is lower than that of TLS. The present case report highlights both entities as possible complications of a relatively common and slow- 


\section{Additional Information}

\section{Disclosures}

Human subjects: Consent was obtained or waived by all participants in this study. Conflicts of interest: In compliance with the ICMJE uniform disclosure form, all authors declare the following: Payment/services info: All authors have declared that no financial support was received from any organization for the submitted work. Financial relationships: All authors have declared that they have no financial relationships at present or within the previous three years with any organizations that might have an interest in the submitted work. Other relationships: All authors have declared that there are no other relationships or activities that could appear to have influenced the submitted work.

\section{References}

1. McGhee-Jez A, Batra V, Sunder T, Rizk S: Spontaneous tumor lysis syndrome as presenting sign of metastatic prostate cancer. Cureus. 2018, 10:e3706. 10.7759/cureus.3706

2. Findakly D, Wang J: Intricate interplay of entwined metabolic and inflammatory life-threatening processes in tumor lysis syndrome complicating prostate cancer: a systematic review with a single institution experience. Cureus. 2020, 12:e7395. 10.7759/cureus.7395

3. Cohen PR, Prieto VG, Kurzrock R: Tumor lysis syndrome: introduction of a cutaneous variant and a new classification system. Cureus. 2021, 13:e13816. 10.7759/cureus.13816

4. Kalter JA, Allen J, Yang Y, Willing T, Evans E: Spontaneous tumor lysis syndrome in an adenocarcinoma of unknown origin. Cureus. 2020, 12:e12169. 10.7759/cureus.12169

5. Kollathodi SB, Parameswaran KK, Madhavan L, Kuruvilla S: Hematological malignancies presenting as spontaneous tumor lysis syndrome: a case series. J Family Med Prim Care. 2018, 7:1116-9. 10.4103/jfmpc.jfmpc_171_18

6. Iftikhar S, Khokher W, Gekonde J, Kesireddy N, Mudiyanselage P: Rituxan-induced tumor lysis syndrome in a patient with diffuse large B-cell lymphoma. Cureus. 2021, 13:e16921. 10.7759/cureus.16921

7. Caravaca-Fontán F, Martínez-Sáez O, Pampa-Saico S, Olmedo ME, Gomis A, Garrido P: Tumor lysis syndrome in solid tumors: clinical characteristics and prognosis. Med Clin (Barc). 2017, 148:121-4. 10.1016/j.medcli.2016.10.040

8. Kekre N, Djordjevic B, Touchie C: Spontaneous tumour lysis syndrome. CMAJ. 2012, 184:913-6. 10.1503/cmaj.111251

9. Gemici C: Tumour lysis syndrome in solid tumours . Clin Oncol (R Coll Radiol). 2006, 18:773-80. 10.1016/j.clon.2006.09.005

10. Mirrakhimov AE, Ali AM, Khan M, Barbaryan A: Tumor lysis syndrome in solid tumors: an up to date review of the literature. Rare Tumors. 2014, 6:5389. 10.4081/rt.2014.5389

11. Myint PT, Butt HW, Alrifai T, Marin C: Spontaneous tumor lysis syndrome secondary to small-cell neuroendocrine carcinoma of unknown origin: a rare case report and literature review. Case Rep Oncol Med. 2019, 2019:6375693. 10.1155/2019/6375693 